\title{
Symptoms and Their Interpretation in Patients Self-Treating Abdominal Cramping and Pain with a Spasmolytic (Butylscopolamine Bromide)
}

\author{
-A Pharmacy-Based Survey
}

\author{
Stefan Müller-Lissner ${ }^{1}$, Eckhard Schäfer ${ }^{2}$, Anke Kondla ${ }^{2}$ \\ ${ }^{1}$ Park-Klinik Weissensee, Charité - Universitätsmedizin Berlin, Germany; ${ }^{2}$ Boehringer Ingelheim, Ingelheim, Germany. \\ Email: mueli@park-klinik.com
}

Received December $13^{\text {th }}$, 2010; revised February $10^{\text {th }}$, 2011; accepted March $1^{\text {st }}, 2011$.

\begin{abstract}
Background: A majority of people suffering from functional abdominal complaints treat themselves with OTC medication. Aim: To gain information on symptoms and their interpretation in patients self-treating abdominal cramping and pain with a spasmolytic (butylscopolamine). Method: 4,680 questionnaires were distributed by 306 pharmacists in Germany to patients suffering from abdominal complaints and buying butylscopolamine. Key findings: Questionnaires from 1,539 respondents were returned, and 1,417 could be evaluated. Abdominal cramping was the major reason for buying butylscopolamine. Only a minority of respondents had consulted a general practitioner (27.3\%) and even less a specialist (12.5\%). Only a minority of subjects met expert criteria for irritable bowel syndrome (13\%). There was a large overlap between self-reported dysmenorrhoea and bowel symptoms (35\%). Conclusions: Real life conditions do not seem to be well covered by expert criteria. Differentiating bowel origin from uterine origin in pelvic pain syndromes seems especially problematic. This may particularly apply to the OTC market.
\end{abstract}

Keywords: Abdominal Pain, Butylscopolamine Bromide, Irritable Bowel Syndrome (IBS), Dysmenorrhoea

\section{Introduction}

Recent market research has shown that up to $46 \%$ of subjects living in the community report abdominal cramping and pain [1]. These are also the most common symptoms of the irritable bowel syndrome (IBS), but IBS is also associated with changes in bowel habits and disordered defecation (diarrhoea/constipation). It has been reported that $20-50 \%$ of all reports of abdominal cramping and pain can be attributed to IBS [2]. This is in line with the prevalence of IBS of $11.5 \%$ reported by Hungin and colleagues, based on a community-based survey of over 40,000 subjects living in several European countries [3]. In Germany, the prevalence of IBS is up to $12.5 \%$ [4]. The majority of sufferers from abdominal cramping and pain or IBS use self-medication with over-the-counter (OTC) drugs to relieve their symptoms [4,5].

One of the medications that can be used to treat abdominal cramping and pain is butylscopolamine butylbromide (from now on abbreviated as butylscopolamine).
This is a quaternary ammonium compound which has antispasmodic effects due to an anticholinergic effect on muscarinic receptors on smooth muscle cells in the gastro-intestinal tract. Butylscopolamine has been registered, in Germany, as a prescription drug for the treatment of IBS symptoms since 1951 and since 2001 also as a nonprescription, or OTC medicine, for the treatment of abdominal cramping pain. A recent survey revealed that up to $15 \%$ of the German sufferers used butylscopola- mine as their main brand to treat abdominal cramping and pain [5]. The efficacy and safety of butylscopola- mine have been demonstrated in several recently re- viewed placebo-controlled clinical studies [6] but few data is available regarding the use of spasmolytics when obtained OTC. Therefore, the purpose of this pharmacy-based patient survey was to gain information on the use of spasmolytics as a self-medication by patients suffering from abdominal cramping and pain in real-life practice (in this case, those who chose self-treatment with butylscopolamine). 


\section{Methods}

\subsection{Study Design}

This was a prospective, open, non-controlled, multi-centre, pharmacy-based, observational, post-marketing survey in patients self-treating their abdominal complaints, presumed by the pharmacist to be caused by IBS, with butylscopolamine (Buscopan ${ }^{\circledR}$ ). From October $15^{\text {th }} 2004$ until April $29^{\text {th }} 2005$, 4680 questionnaires were distributed to pharmacies located all over Germany. Patients had to give written informed consent for their participation in the survey after receiving extensive and comprehensive information.

\subsection{Study Population}

Male and female patients older than 18 years buying butylscopolamine in the pharmacy for self-treatment of abdominal cramping and pain were selected by 306 pharmacists after giving written informed consent. Exclusion criteria were those listed in the summary of product characteristics or labelling available for physicians and the patient information/package insert, namely known hypersensitivity to butylscopolamine or any other component of the product, bowel or urinary tract obstruction, megacolon, myasthenia gravis, narrow angle glaucoma, and tachycardia.

In parallel with the advice given in everyday pharmacist-to-patient consultation when recommending a product for abdominal cramping and pain, the pharmacist asked the interested patients standardised questions, derived from the Rome II-criteria for IBS [7]. The advice of the pharmacist regarding the use of medication for an individual patient was not determined by the study protocol.

The sample size of 1500 returned questionnaires was chosen to detect an adverse event with a probability of $0.1 \%$ with a power of $90 \%$.

\subsection{Treatment}

The butylscopolamine treatment was self-administered and self-paid. More specifically, the patient had to choose an adequate dose suitable for him or herself within the limits stated in the package insert. The recommended single dose intake ranges from $10 \mathrm{mg}$ (1 tablet) to $20 \mathrm{mg}$ (2 tablets) butylscopolamine taken up to three times a day. The maximum recommended daily intake hence is $60 \mathrm{mg}$ (6 tablets) of butylscopolamine.

\subsection{Data Collection}

The data was collected by means of a self-administered patient questionnaire. Patients had to complete the questionnaire on their own (e.g. at home), during or right after termination of the self-medication and had to return it to the pharmacy within 14 days after starting treatment (even if the symptoms were still present). The questionnaire included 30 questions (mainly tick boxes with pre-specified answers) on demographics, actual and usual complaints prompting the intake of butylscopolamine, dosage of butylscopolamine, efficacy of treatment (4-point verbal rating scale (VRS): very good, good, sufficient or bad) including onset and duration of effect as experienced by the patient, occurrence of adverse events, and overall tolerability (4-point VRS: very good, good, sufficient or bad). The questionnaire (in German) is available on request from the corresponding author. The pharmacists sent the completed anonymous questionnaires to the Institute for Medical Informatics, Biometry and Epidemiology (IMIBE) at the University Clinic Essen in Essen, Germany, for analysis. Excluded from the analysis (with the exception of AE reporting) were questionnaires with a completion rate of less than $20 \%$ of all questions, unanswered questions on the treatment effect, or lack of compliance with the inclusion and exclusion criteria.

\subsection{Statistical Methods}

Since this was not a controlled randomized interventional but an observational study all p-values are meant as exploratory and purely descriptive.

The sample was post-hoc divided into three subgroups: group 1: IBS patients; group 2: women with self-reported dysmenorrhoea; and group 3: patients falling into neither of these two groups (in the following labelled as "unspecified abdominal complaints”) (Table 1). Patients were assigned to group 1 if [1] reporting a physician's diagnosis of IBS and not complaining of nausea or menstrual pain or [2] not diagnosed with IBS but suffering from abdominal complaints at least 3 times per month, consisting of abdominal pain, abnormal stool form (constipation, diarrhoea), or distension/bloating but no nausea or menstrual pain. The reason for performing these post-hoc subgroup analyses was that 501 respondents (35\%) reported menstrual pain.

\section{Results}

One hundred eighty five (60\%) pharmacies returned 1539 questionnaires, 1417 fulfilling the requirements for analysis (Figure 1). Females predominated (Table 1). The age distribution was shifted towards younger age groups (Figure 2). Mean age was 40.2 (range 22 - 62) years.

More than $50 \%$ of the respondents suffered from more than one complaint (30\% reported two, 17\% three, and $10 \%$ four and more different symptoms, respectively). Details of the reported complaints are listed in Table 1. 
Table 1. Population characteristics.

\begin{tabular}{|c|c|c|c|c|}
\hline & All respondents & Probable IBS patients & Dysmenorrhoea & Other \\
\hline N (\%) & $1,417(100)$ & $183(12.9)$ & $501(35.4)$ & $733(51.7)$ \\
\hline Mean age (years) & 40.2 & 49.4 & $32.0 *$ & $43.5^{*}$ \\
\hline Female (\%) & $81.0^{1}$ & 74.3 & $99.0^{* 1}$ & $70.4^{1}$ \\
\hline \multicolumn{5}{|l|}{ Complaints (\%) } \\
\hline Abdominal pain & 25.1 & 27.9 & $17.2^{*}$ & 29.7 \\
\hline Abdominal cramping & 45.3 & 53.0 & $34.1^{*}$ & 51.0 \\
\hline Epigastric pain & 13.5 & 18.6 & $4.4^{*}$ & 18.4 \\
\hline Epigastric cramps & 19.9 & 27.3 & $7.0 *$ & 26.9 \\
\hline Menstrual pain & 35.4 & 0.0 & 100.0 & 0.0 \\
\hline Constipation & 3.7 & 12.6 & $0.8^{*}$ & $3.4^{*}$ \\
\hline Diarrhoea & 10.5 & 26.8 & $2.4^{*}$ & $12.0 *$ \\
\hline Flatulence/distended stomach & 14.4 & 50.3 & $3.8^{*}$ & $12.7^{*}$ \\
\hline Feeling bloated & 6.6 & 21.3 & $1.8^{*}$ & $6.3^{*}$ \\
\hline Nausea & 8.5 & 0.0 & $4.2^{*}$ & $13.6^{*}$ \\
\hline
\end{tabular}

${ }^{*} P<0.01$ by Chi-square analysis vs. IBS group. ${ }^{1}$ for 9 patients ( 5 in the dysmenorrhoea and 4 in the "unspecified abdominal complaints" group), sex was not known; therefore, the sum does not add up to 100.
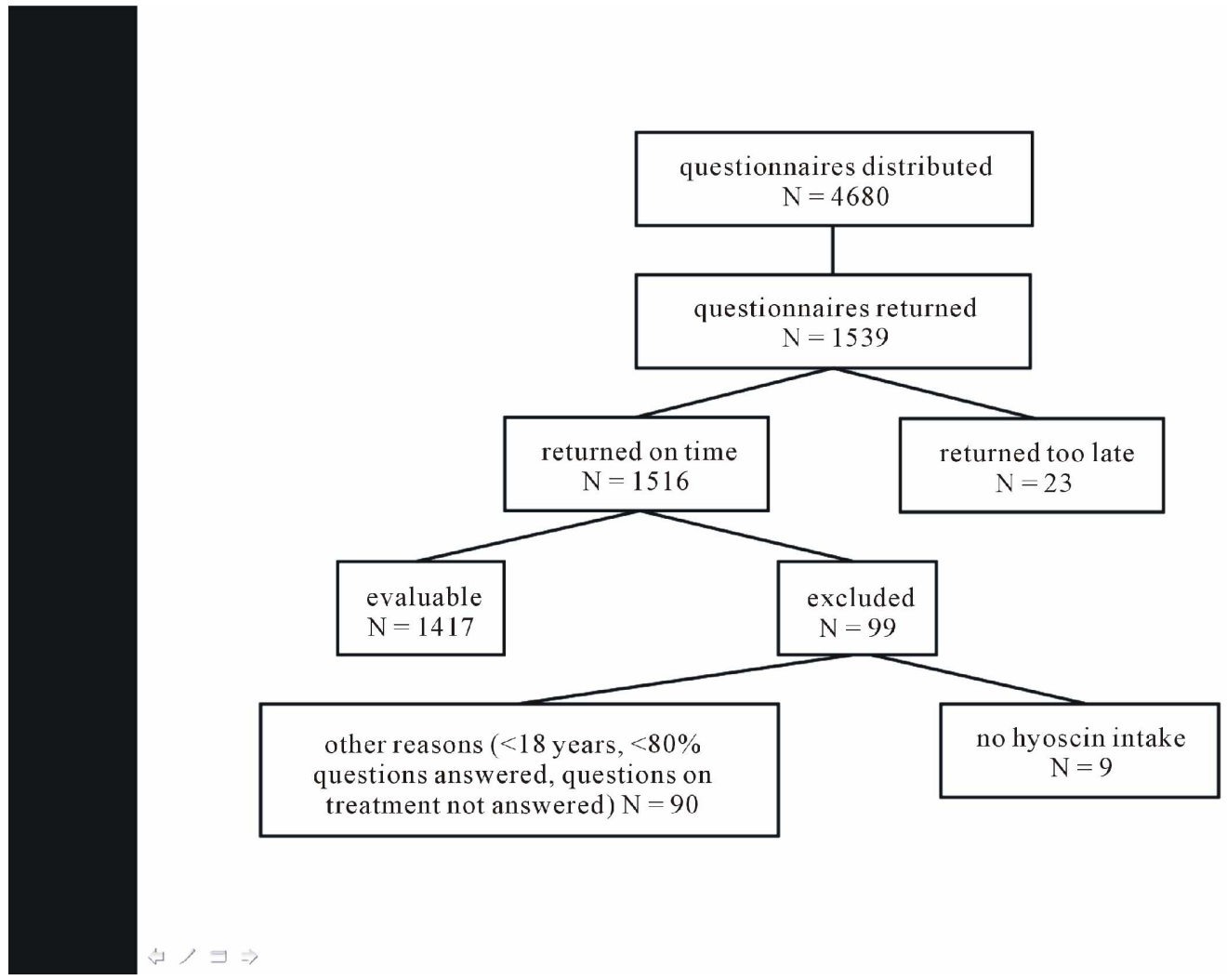

Figure 1. Patient disposition.

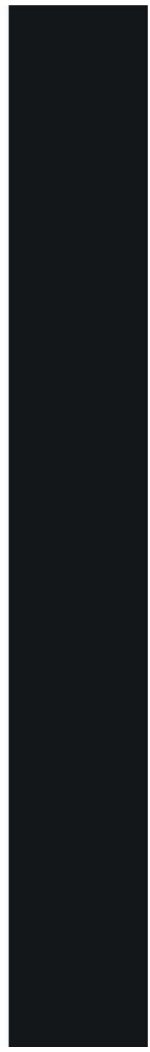




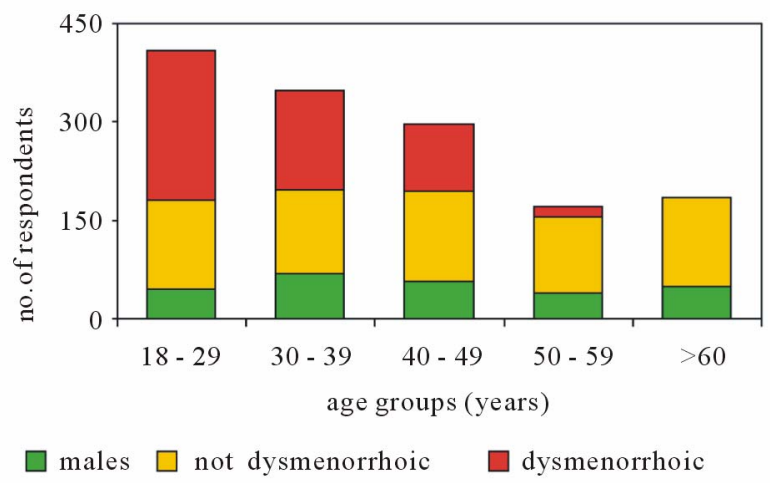

Figure 2. Age distribution among respondents according to subgroup.

The type of complaints in the women with dysmenorrhoea differed by definition considerably from those in the other two groups, with the latter ones being remarkably similar. Bloating was by definition more prevalent in the IBS group (Figure 3).

Two thirds of respondents claimed their abdominal complaints to be severe or very severe and to limit their daily activities without appreciable differences between the three groups. More than $50 \%$ of the participants indicated that their daily and leisure activities were limited by their ailment, and $26 \%$ felt hampered in their professsional activities. Only a minority of respondents had consulted a general practitioner (27.3\%) and even less a specialist (12.5\%).

Two thirds started intake of the spasmolytic within one day of the occurrence of the complaints. Medium duration of a pain attack was 2 days (Figure 4). More than half of dysmenorrhoic women started drug intake at the very beginning of their pain while most subjects from the two other groups waited some time before starting treatment. The dose taken amounted to $1-3$ tablets (mean $3.3 \pm$ 1.5 tablets) for 1 to 5 days (mean $3.2 \pm 3.6$ days). Almost $75 \%$ of the respondents reported to have taken butylscopolamine on demand and $23 \%$ according to a schedule. Most respondents reported that their complaints lasted from 1 to 5 days (mean $3.8 \pm 5.3$ days).

About $80 \%$ of all respondents reported the onset of symptom relief within 45 minutes. Women with dysmenorrhoea experienced a statistically significant faster onset of relief as compared to IBS patients.

Overall efficacy of butylscopolamine on VRS was rated by $87 \%$ of all respondents as "good" or "very good". Although the number of patients rating treatment effects as bad was small $(\mathrm{N}=37)$, perceived treatment failure was most likely among those who delayed initia- tion of treatment for longer than 3 days from the onset of symptoms and who took concomitant medication(s).

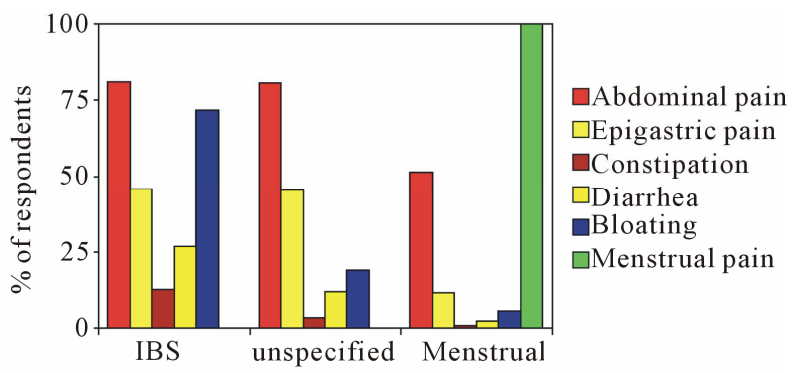

Figure 3. Symptom distribution in the three subgroups.

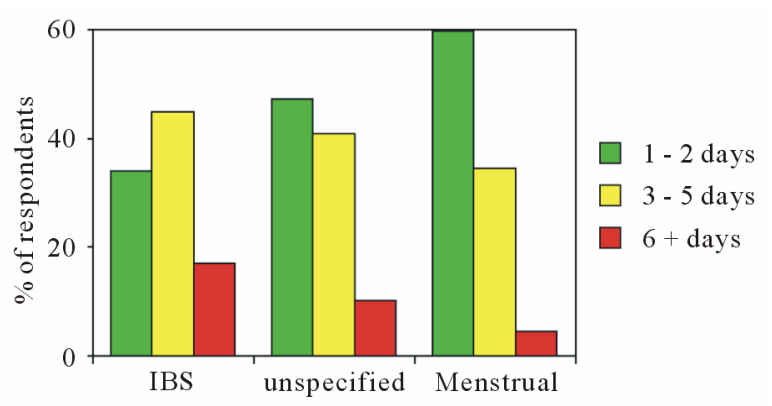

Figure 4. Duration of pain attacks in the three subgroups.

Butylscopolamine was well tolerated with a low incidence of adverse events (in 9.9\% of respondents). Among the adverse events, gastro-intestinal symptoms prevailed (in $1.0 \%$ of respondents). The most commonly reported adverse events included nausea (0.6\%), diarrhoea (0.4\%), dry mouth $(0.4 \%)$, dizziness $(0.3 \%)$, fatigue $(0.3 \%)$, visual disturbance $(0.2 \%)$, and tachycardia $(0.2 \%)$. No serious adverse events or deaths occurred. There was no difference in the frequency of adverse events experienced between subgroups.

\section{Discussion}

This survey was initiated by Boehringer Ingelheim in order to collect information on the population buying butylscopolamine (Buscopan ${ }^{\circledR}$ ) and as such is of limited interest to practising gastroenterologists or gynecologists. However, beyond the scope of this goal interesting results were obtained regarding epidemiology of functional abdominal complaints and their self-treatment with butylscopolamine.

The survey reflects treatment of abdominal complaints in a real-life pharmacy-setting. This approach differs from clinical trials in several aspects. First, diagnoses were not medically confirmed and, therefore, the subgroups contained patients with abdominal cramping and pain, probable IBS and self-reported dysmenorrhoea, respectively. Second, we only looked at patients buying butylscopolamine and patient characteristics could be different for patients buying other self-medications. Third, 
rating treatment efficacy is biased in favour of butylscopolamine as probably only patients satisfied with this treatment will (re)buy this medicine OTC.

On the other hand, restriction of the survey to a group using one and the same drug renders the sample more homogeneous and may allow drawing conclusions with respect to the interpretation of complaints (see below). Obviously, in the chosen uncontrolled setting neither treatment randomisation nor blinding were possible. There fore, we refrain from conclusions regarding efficacy.

Only $13 \%$ of the subjects who visited a pharmacy because of abdominal complaints presumed by the pharmacist to be caused by IBS had IBS according to the criteria as defined in this survey and being close to the Rome criteria [7]. This percentage is somewhat lower than the $20-50 \%$ found in other community-based studies [2] and shows that there are major discrepancies between the classification by a pharmacist and expert criteria. Hence, only a minority of patients with apparently functional lower gut symptoms seem to be covered by the Rome criteria, at least when "diagnosed" by a pharmacist.

In line with previous studies $[1,8,9]$, this survey suggested that more women than men suffer from abdominal complaints (or at least buy butylscopolamine for selftreatment), the ratio being roughly 3:1 to 4:1 (Table 1).

Abdominal cramping and pain show not only a considerable prevalence but do also negatively affect the daily lives of patients in terms of work, sleep, diet, ability to travel, sexual function, as well as personal relationships with family and friends. This has already been observed in previous studies [10-12] and is corroborated by our findings.

Most of the respondents (over 50\%) in the present survey suffered from more than one abdominal complaint. This is not an unexpected finding as co-morbidity exists between IBS and other abdominal symptoms. Of particular interest in this context seems to be dysmenorrhoea. In a previous report $38 \%$ of IBS patients also complained of dysmenorrhoea [13]. Conversely, a diagnosis of IBS was three-fold more common in women with dysmenorrhoea than in those without [14]. In line with these findings, women with dysmenorrhoea constituted a large group among the respondents of this survey (35\%). It has been shown in mechanistic experiments that thresholds for perception and pain in response to rectal balloon distension are lowered in dysmenorrhoic patients [15]. This may contribute to explain the co-occurrence of the two entities. Therefore, a subgroup analysis was performed comparing probable IBS patients to women with selfreported dysmenorrhoea and to patients with other abdominal complaints. Dysmenorrhoic patients had shorter pain attacks than "classical" IBS patients (Figure 4). This could be explained if the lower pain threshold would only be present for a short time period around menses.

Butylscopolamine (alone or in combination with analgesics) has been shown to be effective in treating dysmenorrhoea $[16,17]$ though the quality of these trials is only moderate. At first glance this may not be astonishing since butylscopolamine as a spasmolytic agent relaxes the uterine musculature [18] which is believed to cause the cramping pain in dysmenorrhoea in response to endometrial prostaglandin release [19] though an ischemic nature of the pain via reduced uterine blood flow has also been proposed [20]. However, orally administered butylscopolamine is only minimally absorbed [21] and hence can hardly act on the uterine muscle. For treating intestinal cramps this does not pose a problem since butylscopolamine enters the gut wall from the lumen and inhibits intestinal muscle contractions [22,23]. Its clinical efficacy is independent of the galenic formulation as tablets or capsules [24].

The apparent discrepancy between the positive treatment effect of butylscopolamine in dysmenorrhoea as published previously $[16,17]$ and suggested by our results on one hand and the pharmacological impossibility to act on the uterus on the other hand is-besides a placebo effect-best explained by either a misinterpretation of the origin of pain by the patients ascribing pain to the uterus instead of the bowel or by coexistence of both pain syndromes with considerable relief obtained by calming the bowel. Given the seemingly large overlap between patients with dysmenorrhea and IBS patients, it may be worthwhile to either exclude or specifically treat IBS symptoms in patients suffering from dysmenorrhea in order to more effectively alleviate their symptoms.

\section{Conclusion}

Real life conditions seem somewhat away from the world created by expert criteria. Differentiating bowel origin from uterine origin in lower abdominal pain syndromes seems especially problematic. This may particularly apply to the OTC market. A therapeutic trial with a spasmolytic seems in any case justified.

\section{Acknowledgements}

This research was funded by Boehringer Ingelheim Pharma GmbH \& Co. KG, Ingelheim am Rhein, Germany.

\section{REFERENCES}

[1] E. M. M. Quigley, G. R. Locke, S. Mueller-Lissner, et al., "Prevalence and Management of Abdominal Cramping and Pain: A Multinational Survey," Alimentary Pharmacology and Therapeutics, Vol. 24, No. 2, 2006, pp. 411-419. doi:10.1111/j.1365-2036.2006.02989.x 
[2] D. A. Drossman, Z. Li, E. Andruzzi, et al., "U.S. Householder Survey of Functional Gastrointestinal Disorders," Prevalence, Sociodemography, and Health Impact, Digestive Disease and Sciences, Vol. 38, No. 9, 1993, pp. 1569-1580.

[3] A. P. S. Hungin, P. J. Whorwell, J. Tack and F. Mearin, "The Prevalence, Patterns and Impact of Irritable Bowel Syndrome: An International Survey of 40000 Subjects,” Alimentary Pharmacology and Therapeutics, Vol. 17, 2003, pp. 643-650. doi:10.1046/j.1365-2036.2003.01456.x

[4] A. Icks, B. Haastert, P. Enck, W. Rathmann and G. Giani, "Prevalence of Functional Bowel Disorders and Related Health Care Seeking: A Population-Based Study," Zeitschrift für Gastroenterologie, Vol. 40, No. 3, 2002, pp. 177-183. doi:10.1055/s-2002-22324

[5] S. Mueller-Lissner, E. M. M. Quigley, I. Helfrich and E. Schaefer, "Drug Treatment of Chronic-Intermittent Abdominal Cramping and Pain: A Multi-National Survey on Usage and Attitudes," Alimentary Pharmacology and Therapeutics, Vol. 32, No. 3, 2010, pp. 472-477. doi:10.1111/j.1365-2036.2010.04368.x

[6] G. N. Tytgat, "Hyoscine Butylbromide—A Review of Its Use in the Treatment of Abdominal Cramping and Pain," Drugs, Vol. 67, No. 9, 2008, pp. 1343-1357.

[7] W. G. Thompson, G. F. Longstreth, D. A. Drossman, et al., "Functional Bowel Disorders and Functional Abdominal Pain,” Gut, Vol. 45, Suppl. 2, 1999, pp. 43-47. doi:10.1136/gut.45.2008.ii43

[8] L. Chang, "Review Article: Epidemiology and Quality of Life in Functional Gastrointestinal Disorders,” Alimentary Pharmacology and Therapeutics, Vol. 20, Suppl. 7, 2004, pp. 31-39

[9] F. Mearin, X. Badia, A. Balboa, et al., "Irritable Bowel Syndrome Prevalence Varies Enormously Depending on the Employed Diagnostic Criteria: Comparison of Rome II versus Previous Criteria in a General Population," Scandinavian Journal of Gastroenterology, Vol. 36, No. 11, 2001, pp. 1155-1161.

[10] A. P. S. Hungin, L. Chang, G. R. Locke, E. H. Dennis and V. Barghout, "Irritable Bowel Syndrome in the United States: Prevalence, Symptom Patterns and Impact," Alimentary Pharmacology and Therapeutics, Vol. 21, No. 11, 2005, pp. 1365-1375. doi:10.1111/j.1365-2036.2005.02463.x

[11] M. Proctor and C. Farquhar, "Diagnosis and Management of Dysmenorrhoea,” British Medical Journal, Vol. 332, No. 7550, 2006, pp. 1134-1138. doi:10.1136/bmj.332.7550.1134

[12] D. B. Silk, "Impact of Irritable Bowel Syndrome on Personal Relationships and Working Practices,” European Journal of Gastroenterology, Vol. 13, No. 11, 2001, pp. 1327-1332. doi:10.1097/00042737-200111000-00011

[13] G. Altman, K. C. Cain, S. Motzer, et al., "Increased Symptoms in Female IBS Patients with Dysmenorrhea and PMS,” Gastroenterology Nursing, Vol. 29, No. 1, 2006, pp. 4-11. doi:10.1097/00001610-200601000-00002

[14] M. D. Crowell, N. H. Dubin, J. C. Robinson, et al., "Functional Bowel Disorders in Women with Dysmenorrhea,” American Journal of Gastroenterology, Vol. 89, No. 11, 1994, pp. 1973-1977.

[15] W. Brinkert, G. Dimcevski, L. Arendt-Nielsen, A. M. Drewes and O. H. Wilder-Smith, "Dysmenorrhoea is Associated with Hypersensitivity in the Sigmoid Colon and Rectum,” Pain, Vol. 132, Suppl. 1, 2007, pp. 46-51. doi:10.1016/j.pain.2006.12.011

[16] A. R. de los Santos, R. Zmijanovich, M. S. Perez, M. L. Marti and G. Di Girolamo, "Antispasmodic/Analgesic Associations in Primary Dysmenorrhea Double-Blind Crossover Placebo-Controlled Clinical Trial," International Journal of Clinical Pharmacology Research, Vol. 21 No. 1, 2001, pp. 21-29.

[17] J. H. Kemp, "Buscopan in Spasmodic Dysmenorrhoea," Current Medical Research and Opinion, Vol. 1, No. 1, 1972, pp. 19-25. doi:10.1185/03007997209111141

[18] K. Fujimoto, A. Nakai, T. Okada, T. Ikeuchi, N. Satogami, S. Daido, M. Yakami and K. Togashi, "Effect of Hyoscine Butylbromide (HBB) on the Uterine Corpus: Quantitative Assessment with T2-Weighted (T2W) MRI in Healthy Volunteers,” Journal of Magnetic Resonance Imaging, Vol. 32, No. 2, 2010, pp. 441-445. doi:10.1002/jmri.22252

[19] O. Ylikorkala and M. Y. Dawood, "New Concepts in Dysmenorrhea," American Journal of Obstetrics and Gynecology, Vol. 130, No. 7, 1978, pp. 833-847.

[20] S. Altunyurt, M. Gol, S. Altunyurt, O. Sezer and N. Demir, "Primary Dysmenorrhea and Uterine Blood Flow: A Color Doppler Study," Journal of Reproductive Medicine, Vol. 50, No. 4, 2005, pp. 251-255.

[21] K. Hellstrom, A. Rosen and K. Soderlund, "The Gastrointestinal Absorption and the Excretion of $\mathrm{H3}$ Butylscopolamine (Hyoscine Butylbromide) in Man," Scandinavian Journal of Gastroenterology, Vol. 5, No. 7, 1970, pp. 585-592.

[22] A. R. Pomeroy and M. J. Rand, “Anticholinergic Effects and Passage through the Intestinal Wall of N-Buthylhyoscine Bromide," Journal of Pharmacy and Pharmacology, Vol. 21, No. 3, 1969, pp. 180-187. doi:10.1111/j.2042-7158.1969.tb08224.x

[23] A. Sagrada, A. Schiavone, A. Cefala, P. Trespi and A. Giachetti, "N-Butyl Hyoscine Exerts Local Spasmolytic Effect in the Small and Large Bowel of the Conscious Dog," Archives Internationales de Pharmacodynamie et de Therapie, Vol. 287, No. 2, 1987, pp. 237-247.

[24] Z. Ge, Y. Yuan, S. Zhang, X. Hou, J. Wang, J. Cai, R. Shi, Y. Li, B. Wang and F. Ji, E. Richter and E. Schaefer, "Efficacy and Tolerability of Two Oral Hyoscine Butylbromide Formulations in Chinese Patients with Recurrent Episodes of Self-Reported Gastric or Intestinal SpasmLike Pain,” International Journal of Clinical Pharmacology and Therapeutic, Vol. 49, No. 3, 2011, pp. 198-205. 